\title{
Predicting slow structural transitions in macromolecular systems: Conformational flooding
}

\author{
Helmut Grubmüller* \\ Institut für Medizinische Optik, Theoretische Biophysik, Ludwig-Maximilians-Universität München, \\ Theresienstraße 37, D-80333 München, Germany
}

(Received 3 May 1995)

\begin{abstract}
We present a method to predict complex structural (conformational) transitions in irregular or disordered macromolecular systems, such as proteins or glasses, at the atomic level. Our method aims at rare events, which currently cannot be predicted with traditional molecular dynamics (MD) simulations, since these currently are limited to time scales shorter than a few nanoseconds. Given an initial conformation of the system, our method identifies one or more product states, which may be separated from the initial state by free energy barriers that are large on the scale of thermal energy. It also provides an approximate reaction path, which can be used to determine barrier heights or reaction rates with the usual techniques. The method employs an artificial potential that destabilizes the initial conformation and, thereby, lowers free energy barriers of structural transitions. As a result, transitions are accelerated and may be observed in MD simulations. An analytical estimate for the acceleration factor is given. The method is applied to two test systems, an argon microcluster and a simplified protein model. By these studies we demonstrated that our method is capable of shortening mean transition times from $0.5 \mu \mathrm{s}$ (argon cluster) and $1.4 \mathrm{~ns}$ (protein model) to a few picoseconds. These results suggest that our method is particularly well suited to study biochemically relevant conformational motions in proteins at a microsecond time scale.
\end{abstract}

PACS number(s): 87.15.He, 82.20.Fd, 87.15.By, 82.20.Wt

\section{INTRODUCTION}

Structural transitions are observed in virtually all macromolecular systems. In polymers they determine mechanical, thermodynamic, and transport properties; in fluids and glasses they represent the microscopic basis for structural relaxation. In biological macromolecules like proteins transitions between distinct structural (conformational) states have been observed $[1,2]$.

The latter are of particular interest, since conformational motions in a protein - often fine-tuned at an atomic level - can control, enable, or constitute its specific biochemical function. Well-known examples are functionally important motions in myoglobin [3], the opening and closing of ion channels such as gramicidin [4], allosteric interactions [5], $T \rightleftharpoons R$ transitions [5], conformational transitions in sensory proteins, which initiate signal transduction via $G$ proteins [6], or protein folding processes (for a review on the latter subject see, e.g., Ref. [7]). Recently, pathogenic conformational changes in prion proteins have gained attention as a possible cause of scrapie, "mad cow disease," Creutzfeldt-Jakob disease, and related degeneracies of the nervous system [8].

Proteins exhibit a large number of conformational substates [9] which, when grouped according to transition times or barrier heights, turn out to be hierarchically clustered [10]. Theoretical studies [11-15] agree with

\footnotetext{
*Electronic address:

Helmut.Grubmueller@Physik.uni-muenchen.de
}

these experimental findings (for a review see, e.g., [16]). As a consequence of this hierarchical structure transition times between substates cover a wide range of time scales, from picoseconds $[14,15]$ to years [8].

Conformational transitions (CT's) between substates manifest themselves in sudden changes of tertiary structure. Some CT's are localized (e.g., isomerizations of single bonds inducing "ring flips" [17]), while others involve complex and concerted rearrangements of many atoms in a protein. The above examples are of the latter type.

In this work we will treat CT's in a general framework, but focus on collective CT's. Mainly due to entropic barriers, these are generally slow in terms of transition rates and occur on time scales above nanoseconds. However, an actual event of barrier crossing may be as fast as a few picoseconds. For a study of CT's one has (a) to search for distinct low-energy conformations, (b) to find reaction paths connecting the conformations in configuration space, and (c) to estimate transition rates or mean transition times.

Protein models at atomic resolution, based on and combined with an increasing amount of experimental data on protein structure and dynamics, serve to elucidate microscopic details of protein motion as well as their functional relevance. In particular, molecular dynamics (MD) simulations [18] provide a conceptually straightforward approach. That method is of limited applicability, however, due to the huge computational effort involved. Accordingly, at present only processes faster than a few nanoseconds can be studied that way [19], and complex CT's are usually not observed.

Therefore specialized methods are employed to address 
the three tasks mentioned above. For small systems, adiabatic mapping [20], mode following [21], variable step MD [22], conjugate peak refinement [23], anti-force-bias Monte Carlo methods [24], as well as various other techniques $[25,26]$ have been successfully applied to carry out tasks (a) and (b); a variety of transition state approaches (for a review see, e.g., Refs. $[27,28]$ ) have been used to compute transition rates.

More recently, various methods have been developed to address tasks (b) and (c) also for systems as large as proteins: Given educt and product conformations, path energy minimization [29], the self penalty walk method [30], targeted MD [31], directed dynamics [32], and minimum biasing MD [33] have been applied to determine reaction paths. Once a reaction coordinate has been defined, the method of reactive flux [34], umbrella sampling techniques [35], or thermodynamic perturbation and integration procedures [36-38] can serve to estimate transition rates as well as free energy profiles along the reaction coordinate.

We want to develop a method that allows one to predict CT's without a priori knowledge of reaction paths or product states, i.e., we want to address task (a) for large systems. That method, which we will term "conformational flooding" (CF), should allow us to answer the question: Given an educt conformation (e.g., a structure determined from x-ray or NMR experiments), what slow structural rearrangements can be expected?

Despite their limitations, MD simulations could serve to answer the above question if one could manage to accelerate the CT's during a simulation. One way to achieve this is to raise the temperature, thereby accelerating activated processes by the Arrhenius factor. However, high-temperature MD [39] does not describe a protein under physiological conditions; particularly, that method overestimates the entropic contributions $T \Delta S$ to free energies. It is far from clear whether the CT's observed at high temperatures would also be observed at room temperature.

Thus, we want to accelerate the CT's without seriously affecting free energies of barriers or product states. To that end we will destabilize exclusively the educt state with respect to all other conformational states by modifying and generalizing the umbrella sampling idea [35]: We intend to derive a coarse-grained description of the educt state from short MD simulations, from which we will construct an artificial potential. This potential will be included in the Hamiltonian. It serves to destabilize the educt state and thus should accelerate CT's. If the acceleration factor is large enough, slow CT's may be observed within relatively short MD simulations.

As opposed to conventional umbrella sampling techniques, $C F$ will not require assumptions on reaction paths or product states and, therefore, should allow unbiased prediction of atomic rearrangements. With respect to the substate model which we will employ, CF can also be conceived as a generalization of a class of techniques to compute absolute free energies [40], which were motivated by density functional methods.

The following section first introduces notions and assumptions based on which we then describe our method.
Subsequently, analytical estimates for the expected acceleration of CT's due to our method will be derived. In Sec. III we provide sample applications for two test systems, an argon microcluster and a simplified protein model. Both applications will serve to illustrate the method as well as to compare the estimated acceleration with explicit MD simulations. To enable such comparisons, we had to choose test systems, which are less complex than typical proteins. However, work on a globular protein bovine pancreatic trypsin inhibitor (BPTI) and on an ion channel protein (gramicidin $A$ ) is in progress and will be reported elsewhere.

Conformational flooding has a variety of applications in several fields, e.g., as a tool for protein structure determination or conformational search, to check the stability of protein models, to predict functional motions, or to improve estimates of thermodynamic quantities such as free energies and entropies for proteins, polymers, or glasses. We will sketch these applications in Sec. IV.

\section{THEORY}

\section{A. The subcanonical ensemble}

We start by defining a "conformational substate" [41] (CS) $S_{k}$ of a classical many particle system with Hamiltonian $\mathcal{H}$ as a confined region in phase space, in which the system stays for a while. More precisely, the system is required to stay in that region for a period $T_{0}$ which is long enough to allow the definition of statistical averages of that substate such as average structure, average energy, temperature, etc. The statistical ensemble, from which these thermodynamic averages are derived, will be referred to as the "subcanonical ensemble," and the averages will be denoted by \langle\rangle$_{S_{k}}$.

A consequence of the above definition is the notion of CS's as regions of low free energy $F$ in a properly defined subspace of configuration space [43]. These regions are separated from each other by free energy barriers $\Delta F$. [In this paper, for brevity of notation, we consider the (Helmholtz) free energy $F$ describing constant volume conditions instead of the - physiologically more adequate - (Gibbs) free energy $G$ which refers to constant pressure systems. If needed, the latter can be introduced; see, e.g., Ref. [42].] We note that, since $\Delta F=\Delta U-T \Delta S$, these barriers may originate from barriers in potential energy $\Delta U$ or, at finite temperature $T$, from entropy wells $\Delta S$, or from a combination of both. All these variants have been observed in proteins [44-46] and, therefore, both energy contributions will be considered in our treatment.

The potential energy landscape of proteins is quite complex $[11,12]$, and so is the free energy landscape. To reduce that complexity, we develop an effective, coarsegrained description with an adjustable level of coarse graining. We will proceed in three steps. First, we will introduce the notion of "conformation space" as a subspace in configuration space. On this subspace we will consider a free energy landscape. Second, we will motivate a proper choice of linear collective coordinates. Third, these "conformational coordinates" will serve to 
construct a substate model in terms of an effective Hamiltonian.

\section{B. Free energy in conformation space}

In the canonical ensemble, i.e., for constant temperature $T=1 /\left(k_{B} \beta\right)$, the phase space density $\rho$ is given by the Boltzmann factor,

$$
\rho(\mathbf{x}, \mathbf{p})=Z^{-1} \exp [-\beta \mathcal{H}(\mathbf{x}, \mathbf{p})]
$$

with the partition function

$$
Z:=\int d^{3 N} x d^{3 N} p \exp [-\beta \mathcal{H}(\mathbf{x}, \mathbf{p})]
$$

where $\mathbf{x}$ is the $3 N$-dimensional (Cartesian) vector of the $N$ particle positions which define the configuration of the system, and $\mathbf{p}$ denotes their momenta. For conservative systems the above integral factorizes into configurational and momentum parts. The latter can be eliminated from an explicit description by considering the configuration space density $\rho^{\mathbf{x}}$ :

$$
\rho^{\mathbf{x}}(\mathbf{x})=\int d^{3 N} p \rho(\mathbf{x}, \mathbf{p})
$$

Similarly, one obtains a coarse-grained description of $\rho^{\mathbf{x}}$ by excluding a number $3 N-m(0 \leq m \leq 3 N)$ of configurational (not necessarily Cartesian) degrees of freedom which are assumed not to be involved in CT's, leaving $m$ "important" [47], "conformational" [48], "relevant" [49], or "essential" [50] degrees of freedom $\left(c_{1}, \ldots, c_{m}\right)^{T} \equiv \mathbf{c}=\mathbf{f}(\mathbf{x})$, which we will specify below. On the subspace of the $c_{i}$ we define a "conformation space density" $\rho^{\mathbf{c}}(\mathbf{c})$ as the projected configuration space density [15]

$$
\rho^{\mathbf{c}}(\mathbf{c})=\int d^{3 N} x^{\prime} \rho^{\mathbf{x}}\left(\mathbf{x}^{\prime}\right) \delta\left[\mathbf{c}-\mathbf{f}\left(\mathbf{x}^{\prime}\right)\right]
$$

from which we derive a free energy landscape $F(\mathbf{c})$,

$$
F(\mathbf{c})=-\beta^{-1} \ln \rho^{\mathbf{c}}(\mathbf{c}) .
$$

Equations (1) - (5) refer to ergodic systems and equilibrium conditions; the molecular dynamics of proteins, however, is essentially nonergodic at all time scales (cf. the Levinthal paradox [51]), particularly at the time scales covered by MD simulations. Thus it is necessary to consider the time-dependent conformation space density $\rho^{\mathbf{c}}\left(t, \mathbf{c} \mid t_{0}, \mathbf{c}_{0}\right)$. Being not completely rigorous in our notation (for a rigorous treatment see, e.g., [52]), $\rho^{\mathbf{c}}\left(t, \mathbf{c} \mid t_{0}, \mathbf{c}_{0}\right)$ can be defined as the conditional probability of finding the system in a phase space element $\Delta \rho^{\mathbf{c}}$ at position $c$ and time $t$, given it has been near $c_{0}$ at time $t_{0}$.

In the substate scenario, starting at $t_{0}$ and $\mathbf{c}_{0} \in S_{k}$ and proceeding in time, the region of nonzero $\rho^{\mathbf{c}}\left(t, \mathbf{c} \mid t_{0}, \mathbf{c}_{0}\right)$ will quickly expand within $S_{k}$. At the larger time scale of transition times $T_{0}, \rho^{\mathbf{c}}\left(t, \mathbf{c} \mid t_{0}, \mathbf{c}_{0}\right)$ will gradually "leak" out of $S_{k}$ and "flow" into other substates. If the substates cluster hierarchically according to mean transition times between substates, the same picture applies to transitions at higher hierarchy levels, i.e., to transitions between groups of substates.

Eventually, a range $t_{\min } \leq \Delta t \leq t_{\max }$ exists, for which $\rho^{\mathbf{c}}\left(t_{0}+\Delta t, \mathbf{c} \mid t_{0}, \mathbf{c}_{0}\right)$ is nearly constant and independent of $\mathbf{c}_{0} \in S_{k}$. We will denote this quasistable density as the "conformation density $\rho_{k}^{\mathbf{c}}(\mathbf{c})$ of substate $S_{k}$," which defines subcanonical averages \langle\rangle$_{S_{k}}$.

The alert reader may have noted that the upper scenario is that of well separated time scales. It is well known, though, that in irregular systems time scales are often not well separated [53]. As we cannot treat these cases with rigor, we adopt a pragmatic point of view and define $\rho_{k}^{\mathbf{c}}(\mathbf{c}):=\rho^{\mathbf{c}}\left(t_{0}+\Delta t, \mathbf{c} \mid t_{0}, \mathbf{c}_{0}\right)$ for reasonably (and typically heuristically) chosen $\Delta t$ and $\mathrm{c}_{0} \in S_{k}$. In this work, MD simulations shall serve to characterize $\rho_{k}^{\mathbf{c}}$.

\section{Conformational coordinates, effective Hamiltonian}

To derive a coarse-grained description of CS's we will define an effective Hamiltonian [54], $\mathcal{H}_{\mathrm{eff}}^{k}(\mathbf{c})$ as that potential which would generate, according to Eqs. (1) and (4), an approximate conformation density $\tilde{\rho}_{k}^{\mathbf{c}}(\mathbf{c})$, which serves as a model for $\rho_{k}^{\mathbf{c}}(\mathbf{c})$. The key here is to find appropriate conformational coordinates $c_{i}$ and a density model $\tilde{\rho}_{k}^{\mathbf{c}}$ which is, on the one hand, sufficiently simple to allow an analytical treatment and, on the other hand, characterizes $S_{k}$ well enough. Inspired by the results in Refs. [14,50,43], and in a similar spirit as in Ref. [55] as well as implicitly assumed in Refs. [56,57], we first approximate the configuration density $\rho_{k}^{\mathbf{x}}(\mathbf{x})$ by a multivariate Gaussian centered at $\overline{\mathbf{x}}$,

$$
\rho_{k}^{\mathbf{x}}(\mathbf{x}) \approx \tilde{\rho}_{k}^{\mathbf{x}}(\mathbf{x}):=\tilde{Z}^{-1} \exp \left[-\frac{1}{2}(\mathbf{x}-\overline{\mathbf{x}})^{T} \mathbf{A}(\mathbf{x}-\overline{\mathbf{x}})\right]
$$

with partition function

$$
\tilde{Z}:=\int d^{3 N} x \exp \left[-\frac{1}{2}(\mathbf{x}-\overline{\mathbf{x}})^{T} \mathbf{A}(\mathbf{x}-\overline{\mathbf{x}})\right] .
$$

A $\in \mathcal{R}^{3 N \times 3 N}$ is a symmetric, positive semidefinite matrix, which defines the shape of the Gaussian. It is derived from the subcanonical covariance matrix $\mathbf{C}$ [52],

$$
\mathbf{A}^{-1}=\mathbf{C}:=\left\langle(\mathbf{x}-\overline{\mathbf{x}})(\mathbf{x}-\overline{\mathbf{x}})^{T}\right\rangle_{S_{k}}
$$

with

$$
\overline{\mathbf{x}}=\langle\mathbf{x}\rangle_{S_{k}}
$$

$\mathbf{C}$ and $\overline{\mathbf{x}}$ are computed from MD simulations. (For the treatment of the six eigenvectors which describe rigid body motions see Sec. III A 1.)

Diagonalizing $\mathbf{A}=\mathbf{Q}^{T} \mathbf{\Lambda} \mathbf{Q}$ with orthonormal $\mathbf{Q} \in$ $\mathcal{R}^{3 N \times 3 N}$ and diagonal $\boldsymbol{\Lambda}=\left(\delta_{i j} \lambda_{i}\right)_{i, j=1, \ldots, 3 N}$ yields collective coordinates $\mathbf{q}=\mathbf{Q}(\mathbf{x}-\overline{\mathbf{x}})$, which serve to simplify Eq. (6):

$$
\tilde{\rho}_{k}^{\mathbf{x}}=\tilde{Z}^{-1} \exp \left[-\frac{1}{2} \mathbf{q}^{T} \mathbf{\Lambda} \mathbf{q}\right]
$$


For our coarse grained description we select the $m$ collective coordinates $\mathbf{c}=\left(q_{1}, \ldots, q_{m}\right)^{T}$ with smallest eigenvalues $\lambda_{i}$. This choice is based on the observation that the remaining $q_{i}$ with large eigenvalues describe localized, high-frequency, nearly harmonic vibrational modes with small amplitudes which, therefore, are not expected to reflect CT's. In contrast, the dynamics of the $m$ conformational coordinates $c_{i}$ is slow, essentially anharmonic, and it is known to dominate the collective motions in proteins $[58,14,43]$. We therefore assume that our choice captures the relevant degrees of freedom for CT's. The number $m$ of conformational degrees of freedom which are explicitly considered determines the level of coarsegraining.

Inserting $\tilde{\rho}_{k}^{\mathbf{x}}$ into Eq. (4) defines our conformation density model,

$$
\tilde{\rho}_{k}^{\mathbf{c}}(\mathbf{c}):=\tilde{Z}_{c}^{-1} \exp \left[-\frac{1}{2} \mathbf{c}^{T} \boldsymbol{\Lambda}_{c} \mathbf{c}\right]
$$

where $\boldsymbol{\Lambda}_{c}=\left(\delta_{i j} \lambda_{i}\right)_{i, j=1, \ldots, m}$, and $\tilde{Z}_{c}$ is the appropriate partition function. From this density model, with Eq. (1), the effective Hamiltonian $\mathcal{H}_{\text {eff }}^{k}(\mathbf{c})$ for substate $S_{k}$ reads

$$
\mathcal{H}_{\mathrm{eff}}^{k}(\mathbf{c})=\frac{1}{2} \beta^{-1} \mathbf{c}^{T} \boldsymbol{\Lambda}_{c} \mathbf{c}
$$

It is instructive to consider a few properties of $\mathcal{H}_{\text {eff }}^{k}$. By definition, $\mathcal{H}_{\text {eff }}^{k}$ is harmonic. In particular, for systems with harmonic potential $V(\mathbf{x})$ we have $\mathcal{H}_{\text {eff }}^{k}(\mathbf{c})=$ $V\left(\mathbf{Q}^{T} \mathbf{c}\right)+$ const (where only the first $m$ columns of $\mathbf{Q}^{T}$ are used). In the realistic case of highly nonlinear systems, however, the relation between $\mathcal{H}_{\text {eff }}^{k}$ and $V$ is complicated. Figure 1 depicts a sketch of two limiting cases. If entropic contributions to the free energy are small [Fig. 1(a)], then $\mathcal{H}_{\text {eff }}^{k}$ (bold line) represents a smoothed approximation to $V$ (thin, solid line) within $S_{k}$; if entropic contributions are large compared to variations in $V, \mathcal{H}_{\text {eff }}^{k}$ is not necessarily (nor is it intended to be) a good approximation to $V$, and Fig. 1(b) provides a

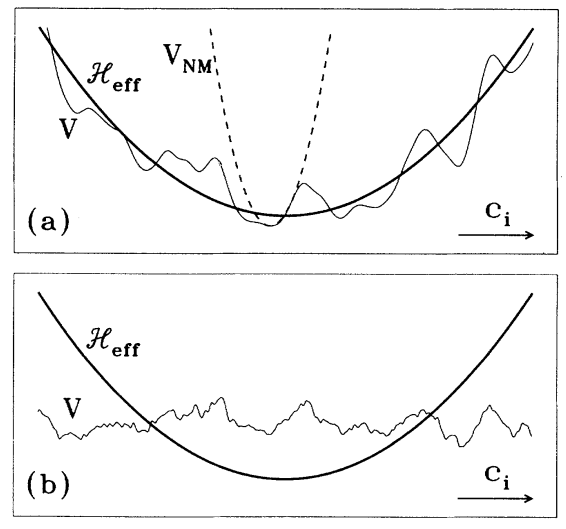

FIG. 1. Sketch of potential energy $V$ (c) (thin, solid lines), $\mathcal{H}_{\text {eff }}^{k}(\mathrm{c})$ (bold), and harmonic approximations $V_{\mathrm{NM}}(\mathbf{c})$, as used in normal mode analysis (dashed); the two pictures refer to (potential) energy (a) and entropy (b) dominated situations, respectively. more adequate representation of the situation (the onedimensional sketch in the figure lacks a representation of entropic barriers, since the latter arise from the high dimensionality and a probably "mazelike" structure of the energy landscape).

We also wish to comment on the relation between $\mathcal{H}_{\text {eff }}^{k}$ and a second order Taylor expansion $V_{\mathrm{NM}}$ of the potential energy at some energy minimum [dashed line in Fig. 1(a)], which is studied in normal mode analysis. As indicated in the figure, and due to the multiminimum structure of the energy landscape, $\mathcal{H}_{\text {eff }}^{k}(\mathbf{c})$ generally differs from $V_{\mathrm{NM}}\left(\mathbf{Q}^{T} \mathbf{c}\right)$. This is particularly the case for proteins at physiological temperatures [59]. By definition, $V_{\mathrm{NM}}$ is a local description of $V$, whereas $\mathcal{H}_{\text {eff }}^{k}$ characterizes the system at the mesoscopic scale of a particular substate. For that reason we consider $\mathcal{H}_{\text {eff }}^{k}$ a more appropriate description for CS's.

\section{Conformational flooding}

The coarse-grained substate model $\mathcal{H}_{\text {eff }}^{k}$ provides the basis to design a "flooding" potential $V_{\mathrm{ff}}(\mathbf{c})$, which is to be included into $\mathcal{H}(\mathbf{x}, \mathbf{p})$ during $\mathrm{MD}$ simulations, and which is supposed to accelerate CT's. In agreement with our assumption that the conformational coordinates $c_{i}$ describe CT's sufficiently accurately, we define the flooding potential as a function of only these $m$ degrees of freedom.

Qualitatively, we intend to modify the free energy landscape $F$ as indicated in Fig. 2. In the figure, the bold line represents $F(c)$ in the vicinity of a substate $S_{k}$ (well) as a function of one particular $c_{i}$. Also shown is a free energy barrier separating $S_{k}$ from other substates (which are not shown). The purpose of the flooding potential $V_{\mathrm{fl}}$ is to raise the free energy within $S_{k}$ (thin line) so as to destabilize that initial substate and to drive the system into another substate. As is also indicated in the figure, we require $V_{\mathrm{ff}}$ to be short ranged, so that the barrier is unaffected. With that assumption, the free energy barrier height is reduced by an amount $\Delta F$ indicated in the figure and defined below, and one expects a corre-

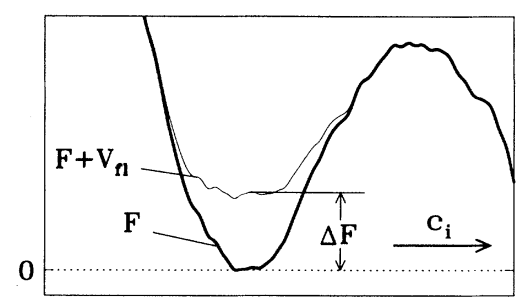

FIG. 2. "Conformational flooding" lowers free energy barriers of CT's and thus should accelerate the transitions. The figure shows a cut through the free energy landscape $F\left(c_{i}\right)$ (bold line) along a particular conformational coordinate $c_{i}$ in the vicinity of a CS (well). To the right, a free energy barrier separates the substate from another one (not shown). Inclusion of the artificial flooding potential $V_{\mathrm{f}}$ into the Hamiltonian of the system reduces the barrier height by an amount $\Delta F$ (thin line). 
sponding acceleration of CT's. That process is termed "conformational flooding."

For a proper choice of the flooding potential we require two criteria to be fulfilled, which ensure that $V_{\mathrm{fl}}$ "fits" into $S_{k}$.

(1) Locality. Only the initial substate $S_{k}$ should be affected by the artificial flooding potential, i.e., $V_{f}$ has to vanish outside $S_{k}$. We particularly require $V_{\mathrm{fl}}$ to be small (with respect to $k_{B} T$ ) within the transition region of the CT under consideration.

(2) Uniformity. Within $S_{k}, V_{\mathrm{fl}}$ should be smooth and nonzero everywhere. This ensures that the system will not "hide" in a niche within $S_{k}$ and stay there, unaffected by the flooding potential.

To meet these requirements we chose a (multivariate) Gaussian,

$$
V_{\mathrm{fl}}(\mathbf{c}):=E_{\mathrm{fl}} \exp \left[-\frac{1}{2} \mathbf{c}^{T} \boldsymbol{\Lambda}_{\mathrm{fl}} \mathbf{c}\right]
$$

where $E_{\mathrm{fl}}$ is the strength of the flooding potential and $\boldsymbol{\Lambda}_{\mathrm{fl}}$ describes its shape in conformation space (this is an arbitrary choice; many other smooth, localized functions would do equally well). To "fit" the flooding potential into the harmonic effective Hamiltonian $\mathcal{H}_{\text {eff }}^{k}$ we require the principal axes of $V_{\mathrm{fl}}$ to be parallel to those of $\mathcal{H}_{\mathrm{eff}}^{k}$, and the widths of $V_{\mathrm{fl}}$ along these axes are set proportional to those of $\mathcal{H}_{\text {eff }}^{k}$, i.e.,

$$
\boldsymbol{\Lambda}_{\mathrm{fl}}:=\boldsymbol{\Lambda}_{c} / \gamma^{2}
$$

The constant of proportionality $\gamma^{2}$ remains to be specified as a function of $V_{\mathrm{fl}}$. To that end consider Fig. 3 which shows the "flooding" (thin, dashed-dotted lines) of a harmonic effective Hamiltonian (bold line) using flooding potentials of varying strength (thin, solid lines). In the figure, the two above criteria, locality and uniformity, are balanced by increasing the width of $V_{\mathrm{fl}}$ with $E_{\mathrm{fl}}$ such that the minimum of $\mathcal{H}_{\mathrm{eff}}^{k}+V_{\mathrm{fl}}$ at the substate center $\overline{\mathbf{c}}$ is quartic, i.e.,

$$
\left.\frac{\partial^{2}\left(\mathcal{H}_{\mathrm{eff}}^{k}+V_{\mathrm{ff}}\right)}{\partial c_{i} \partial c_{j}}\right|_{\mathbf{c}=\overline{\mathbf{c}}}=0 \quad \forall i, j=1, \ldots, m
$$

which specifies $\gamma$ as

$$
\gamma=\sqrt{\beta E_{\mathrm{fl}}} .
$$

As required, our choice of $V_{\mathrm{fl}}$ reduces the depth of the energy well uniformly without extending much into the high-energy regions of conformation space, where barriers are to be expected.

We would like to stress that our flooding potential will not push the system towards any preselected destination in configuration space; hence no bias is included as to which product state the system will move to. Rather, the method is likely to follow transition paths of low free energy and thus should identify those neighboring CS's to which also the unperturbed system $\left(V_{\mathrm{fl}}=0\right)$ would move at much slower time scales.

Additionally, the general form chosen for the flooding potential in terms of collective conformational coordinates allows one to vary the level $m$ of coarse graining.

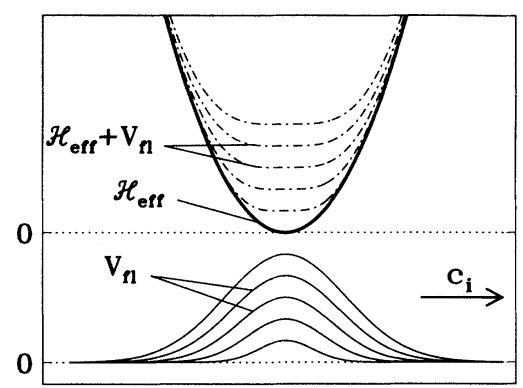

FIG. 3. Harmonic effective Hamiltonian $\mathcal{H}_{\text {eff }}^{k}$ (bold line) and Gaussian-shaped flooding potential $V_{\mathrm{ff}}$ for various flooding strengths $E_{\mathrm{f}}$ (thin, solid lines) as a function of one conformational coordinate $c_{i}$. Adding $V_{\mathrm{f}}$ to the effective Hamiltonian of the system decreases the depth of the substate well (dashed-dotted lines). To meet the criteria of locality and uniformity, the width of $\mathcal{H}_{\text {eff }}^{k}$ is increased with its height as defined by Eq. (16).

In particular, this allows one to "focus" the flooding procedure on those $m$ degrees of freedom which are of interest in a particular application, while leaving the others unaffected.

\section{E. Estimate of transition rates}

What accelerations of CT's for given flooding strength $E_{\mathrm{fl}}$ can be expected? More precisely, we want to estimate the acceleration factor $\alpha:=T_{0} / T_{\mathrm{f}}$, where $T_{\mathrm{fl}}$ and $T_{0}$ are the mean transition times (MTT's) out of the initial substate $S_{k}$ with and without flooding potential applied. With that aim we will first derive the (multidimensional) canonical transition state result [60], which in the case of harmonic well and barrier is known to lead to the Kramers equation [61]. The transition state approximation is based on several assumptions, though, which are generally not fulfilled in our case. Therefore we will subsequently drop two of the assumptions and study the resulting corrections to the transition state result.

The first assumption is that a MTT can be defined. This is strictly true only for systems with a clearcut separation of time scales and is, therefore, not guaranteed here. We circumvent this problem by noting that, given a dividing hypersurface $S_{k}^{\neq}$which separates $S_{k}$ from all other substates, a mean first passage time (MFPT) through that surface can be defined in any case. From the MFPT we will derive an operational - though not rigorous - MTT [see Sec. III A 1].

Secondly, transition state theory requires local equilibrium at all times. This is a very strong assumption, and it may often not be fulfilled. Particularly during fast CT's the system may not be in equilibrium with its environment which acts as a heat bath. Similarly, the onset of the flooding potential perturbs the system, and it may not relax towards equilibrium within a time short compared to $T_{\mathrm{fl}}$. To quantify the influence of such non-equilibrium effects, we will derive corrections to the 
transition state result in Secs. IIF and II G. Readers not interested in these details may prefer to skip these two sections.

Finally, for the sake of obtaining an analytical estimate for the acceleration, we modeled $\rho^{\mathbf{c}}$ by a Gaussian, which we consider a rather crude approximation. Therefore, comparisons of the estimate with computer experiments (given in Secs. III A and IIIB) should provide information on the quality of this simple ansatz.

With these assumptions in mind we write the transition state MTT $T_{0}$ for unperturbed transitions out of $S_{k}$ as [28]

$$
T_{0}=\omega^{-1} \kappa_{0}^{-1} \frac{Z_{0}}{Z_{0}^{\neq}}
$$

where

$$
Z_{0}:=Y_{0} \int d^{m} c \rho_{k}^{\mathbf{c}}(\mathbf{c})
$$

is the subcanonical partition function,

$$
Z_{0}^{\neq}:=Y_{0}^{\neq} \int_{\Delta S_{k}^{\neq}} d^{m} c \rho_{k}^{\mathbf{c}}(\mathbf{c})
$$

is the partition function of the transition region (defined by a small volume element $\Delta S_{k}^{\neq}$containing the dividing hypersurface), $\omega$ is a characteristic frequency of the system, and $\kappa_{0}$ is the transmission coefficient [34]. $Y_{0}$ and $Y_{0}^{\neq}$are the contributions of the fast degrees of freedom $q_{m+1}, \ldots, q_{3 N}$, and of the momenta to the partition functions. Using similar notations, the MTT $T_{\mathrm{f}}$ for transitions accelerated by $V_{\mathrm{fl}}(\mathbf{c})$ reads

$$
T_{\mathrm{fl}}=\omega^{-1} \kappa_{\mathrm{fl}}^{-1} \frac{Z_{\mathrm{fl}}}{Z_{\mathrm{fl}}^{\neq}}
$$

with

$$
Z_{\mathrm{fl}}:=Y_{\mathrm{fl}} \int d^{m} c \rho_{k}^{\mathbf{c}}(\mathbf{c}) \exp \left[-\beta V_{\mathrm{fl}}(\mathbf{c})\right]
$$

and

$$
Z_{\mathrm{fl}}^{\neq}:=Y_{\mathrm{fl}}^{\not \neq} \int_{\Delta S_{k}^{\neq}} d^{m} c \rho_{k}^{\mathbf{c}}(\mathbf{c}) \exp \left[-\beta V_{\mathrm{fl}}(\mathbf{c})\right]
$$

Since, by construction, the flooding potential depends on none of the degrees of freedom described by the $Y$ contributions, we assume the latter to be independent of $V_{\mathrm{fl}}$ and drop these factors below. Then, Eqs. (17) and (20) give the acceleration factor

$$
\alpha:=\frac{T_{0}}{T_{\mathrm{fl}}}=\frac{\kappa_{\mathrm{fl}}}{\kappa_{0}} \frac{Z_{\mathrm{f}}^{\neq}}{Z_{0}^{\neq}} \frac{Z_{0}}{Z_{\mathrm{fl}}} .
$$

Since the flooding potential was required to be small within the transition region (barrier top in Fig. 2), and due to the equilibrium assumption, the dynamics of the system near the dividing surface will be unaffected by $V_{\mathrm{fl}}$, implying $\kappa_{0} \approx \kappa_{\mathrm{f}}$ and $Z_{0}^{\neq} \approx Z_{\mathrm{fl}}^{\neq}$. With that approximation, Eq. (23) reduces to

$$
\alpha \approx \frac{Z_{0}}{Z_{\mathrm{fl}}}
$$

We emphasize that in this transition state approach no specific value for the transmission coefficients $\kappa_{0}$ and $\kappa_{\mathrm{fl}}$ was required, since only rate ratios are considered; similarly, there was no need to specify the dividing surface. Hence, up to Eq. (24), our transition state approach should be reasonably accurate as long as the equilibrium assumption holds.

To arrive at an analytical estimate $\tilde{\alpha}$ for $\alpha$, we have to introduce a crude approximation, though, in that we replace the exact phase space density $\rho_{k}^{\mathbf{c}}$ by the Gaussian density model $\tilde{\rho}_{k}^{\mathbf{c}}$ defined in Eq. (11). From Eq. (24), and inserting $\rho_{k}^{\mathrm{c}}$ into Eqs. (18) and (21), respectively, we obtain the acceleration estimate

$$
\alpha \approx \tilde{\alpha}=\frac{\int d^{m} c \exp \left[-\frac{1}{2} \mathbf{c}^{T} \boldsymbol{\Lambda}_{\mathrm{ff}} \mathbf{c}\right]}{\int d^{m} c \exp \left[-\frac{1}{2} \mathbf{c}^{T} \boldsymbol{\Lambda}_{\mathrm{fl}} \mathbf{c}-\beta V_{\mathrm{fl}}(\mathbf{c})\right]}
$$

Using Eqs. (13), (14), and (16), and substituting $r^{2}=$ $\mathbf{c}^{T} \boldsymbol{\Lambda}_{\mathrm{fl}} \mathbf{c}$, this estimate simplifies to

$$
\tilde{\alpha}=\frac{\int_{0}^{\infty} d r r^{m-1} \exp \left[-r^{2} / 2\right]}{\int_{0}^{\infty} d r r^{m-1} \exp \left[-r^{2} / 2-\varepsilon e^{-r^{2} /(2 \varepsilon)}\right]},
$$

where $\varepsilon:=\beta E_{\mathrm{fl}}$ is the flooding strength in units of $k_{B} T$.

The integral in the denominator of Eq. (26) cannot be solved analytically. For $m \gtrsim 10, \tilde{\alpha}$ may be approximated by replacing $r^{m-1} \exp \left[-r^{2} / 2\right]$ with a Gaussian of width $1 / \sqrt{2}$, centered at $r_{0}=(m-1)^{1 / 2}$, and by expanding $\exp \left[-r^{2} /(2 \varepsilon)\right]$ into a power series up to second order in $r-r_{0}$. Then both integrands in Eq. (26) are Gaussian, and

$$
\ln \tilde{\alpha} \approx \varepsilon \eta-\frac{(m-1) \eta^{2} / 2}{2+a}+\frac{1}{2} \ln (1+a / 2)
$$

with $\eta:=\exp \left[-\frac{1}{2} m / \varepsilon\right]$ and $a:=\eta(m-\varepsilon) / \varepsilon$. Because $0<a<1 / 2$ for $\varepsilon<m-1$, and with $m-1 \approx m$, Eq. (27) reduces to the final result

$$
\ln \tilde{\alpha} \approx \varepsilon \eta-\frac{1}{4} m \eta^{2}+\frac{1}{8} \eta(m / \varepsilon-1)\left(2+m \eta^{2}\right) .
$$

A closer inspection of the three terms in Eq. (28) reveals that the leftmost term, $\varepsilon \eta$, dominates the behavior of the acceleration estimate $\tilde{\alpha}$ (zeroth order approximation); the second term is a $10 \%$ correction, while the third is negligible.

Defining the free energies of activation for CT's with and without flooding potential as $\Delta F_{\mathrm{fl}}^{\neq \neq}:=k_{B} T \ln \left(\omega T_{\mathrm{f}}\right)$ and $\Delta F_{0}^{\neq}:=k_{B} T \ln \left(\omega T_{0}\right)$, respectively, $k_{B} T \ln \alpha=$ $\Delta F_{0}^{\neq}-\Delta F_{\mathrm{f}}^{\neq}$can be interpreted as the destabilization free energy $\Delta F$ due to the flooding potential, and we have

$$
\Delta F^{\mathrm{TS}}=k_{B} T \ln \tilde{\alpha},
$$

where the index denotes the transition state result. The destabilization energy has already been introduced qualitatively in Fig. 2. For later use we note that the right hand side of Eq. (25) approximates (by means of $\tilde{\rho}_{k}^{\mathrm{c}}$ ) the 
subcanonical average

$$
\frac{1}{\tilde{\alpha}} \approx\left\langle e^{-\beta V_{\mathrm{fl}}}\right\rangle_{\tilde{S}_{k}},
$$

and therefore

$$
\Delta F^{\mathrm{TS}} \approx-\beta^{-1} \ln \left\langle e^{-\beta V_{\mathrm{f1}}}\right\rangle_{\tilde{S}_{k}} \approx\left\langle V_{\mathrm{fl}}\right\rangle_{\tilde{S}_{k}}
$$

The latter approximation holds for small fluctuations,

$$
\beta\left[\left\langle\left(\left\langle V_{\mathrm{ff}}\right\rangle_{\tilde{S}_{k}}-V_{\mathrm{ff}}\right)^{2}\right\rangle_{\tilde{S}_{k}}\right]^{1 / 2} \ll 1
$$

which scale as $m^{-1 / 2}$.

It will be convenient to consider energies per degree of freedom $c_{i}$, where we refer only to the $m$ conformational coordinates. With $\epsilon_{\mathrm{fl}}:=E_{\mathrm{fl}} / m$ and $\Delta f:=\Delta F^{\mathrm{TS}} / m$ the leading term in Eq. (28) reads

$$
\Delta f=\epsilon_{\mathrm{fl}} \exp \left(-\frac{1}{2 \epsilon_{\mathrm{fl}}}\right)
$$

and is independent of $m$.

Figure 4 shows the destabilization energy per degree of freedom, $\Delta f$, as a function of flooding strength $\epsilon_{\mathrm{fl}}$, as well as the estimated acceleration $\tilde{\alpha}$ for $m=100$ conformational coordinates. The solid curve has been obtained by numerical integration of Eq. (26); the dashed and dashed-dotted curves represent zeroth and second order approximations [Eqs. (33) and (28)], respectively.

As can be seen from Eq. (33) and also in Fig. 4, $\Delta f$ does not scale linearly with the flooding strength. For $\epsilon_{\mathrm{ff}}<0.1$ the exponential in Eq. (33) is small, and the system does not "see" any flooding; only above values of 0.2 are considerable acceleration rates expected. We draw particular attention to the fact that even for flooding strengths as large as $\epsilon_{\mathrm{fl}}=\mathbf{0 . 4}$, where acceleration factors of up to $10^{4}$ are expected, the artificial free energy increase is small $\left(0.1 k_{B} T\right)$. As a result, the dynamics within the initial CS should be essentially unaffected by the flooding method.

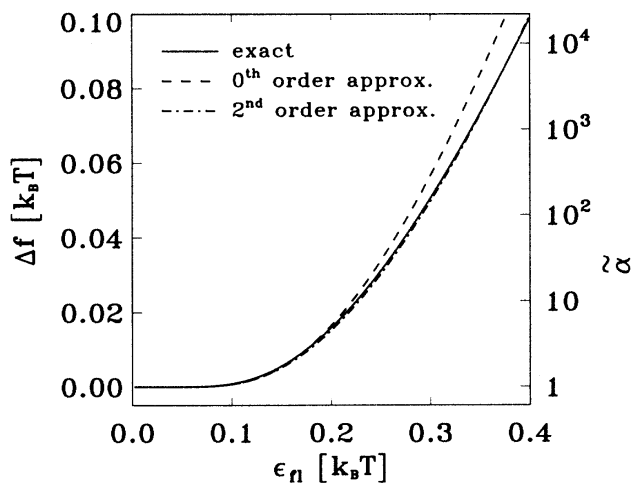

FIG. 4. Destabilization free energy $\Delta f$ per degree of freedom and estimated acceleration factor $\tilde{\alpha}$ as a function of flooding strength $\epsilon_{\mathrm{fl}}$ for $m=100$ degrees of freedom. The four curves have been obtained by numerically integrating Eq. (26) (solid), and by zeroth (dashed) and second (dashed-dotted) order approximations [Eqs. (27) and (28)].

\section{F. Adiabatic correction to Eq. (26)}

To estimate the acceleration factor $\alpha$ [Eq. (26)] we assumed local equilibrium at all times and particularly during CT's, implying $Z_{0}^{\neq} \approx Z_{\mathrm{fl}}^{\neq}$. However, typical CT's are fast processes, such that the system is not likely to be in equilibrium with the heat bath during the transition, and the acceleration estimate has to be corrected properly. To obtain an upper limit for such a correction, we consider the case of no energy exchange between the system and the heat bath, i.e., we treat the CT as an adiabatic process. Accordingly, the kinetic and potential energies within the substate and within the transition region, respectively, obey

$$
\left\langle E_{\text {kin }}\right\rangle_{S_{k}}+\left\langle V+V_{\mathrm{fl}}\right\rangle_{S_{k}}=\left\langle E_{\mathrm{kin}}\right\rangle_{\neq}+\left\langle V+V_{\mathrm{ff}}\right\rangle_{\neq}
$$

Still we assume the $3 N-6$ degrees of freedom of the system (rigid body motion eliminated) to be in equilibrium with each other; thus, due to equipartition,

$$
\left\langle E_{\mathrm{kin}}\right\rangle_{S_{k}}=\frac{1}{2}(3 N-6) / \beta
$$

and

$$
\left\langle E_{\mathrm{kin}}\right\rangle_{\neq}=\frac{1}{2}(3 N-6) / \beta_{\mathrm{fl}}^{\neq}
$$

The latter equation defines a temperature $\left(k_{B} \beta_{\mathrm{fl}}^{\neq}\right)^{-1}$ within the transition region. Similarly we define $\beta_{0}^{\neq}$for unperturbed CT's.

These transient temperatures differ from the equilibrium temperature $1 /\left(k_{B} \beta\right)$, and the corresponding partition functions $Z_{0}^{\neq}$and $Z_{\mathrm{f}}^{\not}$ have to be corrected appropriately due to the contribution of the conformational momenta $p_{i}:=M_{i} \dot{c}_{i}$,

$$
\frac{Z_{\mathrm{fl}}^{\neq}}{Z_{0}^{\neq}}=\frac{\int d^{m} p \exp \left[-\beta_{\mathrm{fl}}^{\neq} \sum_{i=1}^{m} p_{i}^{2} /\left(2 M_{i}\right)\right]}{\int d^{m} p \exp \left[-\beta_{0}^{\neq} \sum_{i=1}^{m} p_{i}^{2} /\left(2 M_{i}\right)\right]}=\left(\frac{\beta_{0}^{\neq}}{\beta_{\mathrm{fl}}^{\neq}}\right)^{\frac{m}{2}}
$$

where the $M_{i}$ are the appropriate reduced masses of the $c_{i}$. Combining Eqs. (34)-(37) gives the correction factor

$$
\frac{Z_{\mathrm{f}}^{\neq}}{Z_{0}^{\neq}}=\left[\frac{(3 N-6) / \beta+\Delta V+\Delta V_{\mathrm{f}}}{(3 N-6) / \beta+\Delta V}\right]^{\frac{m}{2}},
$$

with $\Delta V=\langle V\rangle_{S_{k}}-\langle V\rangle_{\neq}$and $\Delta V_{\mathrm{fl}}=\left\langle V_{\mathrm{fl}}\right\rangle_{S_{k}}-\left\langle V_{\mathrm{ff}}\right\rangle_{\neq}$. Since $V_{\mathrm{ff}}$ is by construction small within the transition region, $\left\langle V_{\mathrm{fl}}\right\rangle_{\neq}=0$, and from Eq. (31) $\Delta V_{\mathrm{fl}} \approx \Delta F^{\mathrm{TS}}$. Furthermore we assume $\Delta V \ll(3 N-6) / \beta$ and obtain

$$
\frac{Z_{\mathrm{f}}^{\neq}}{Z_{0}^{\neq}} \approx\left(1+\frac{\beta \Delta F^{\mathrm{TS}}}{3 N-6}\right)^{\frac{m}{2}} .
$$

Using Eq. (23), this result implies the adiabatic correction 


$$
\Delta F^{\mathrm{ad}}=\beta^{-1} \ln \frac{Z_{\mathrm{f}}^{\neq}}{Z_{0}^{\neq}} \approx \frac{m}{2(3 N-6)} \Delta F^{\mathrm{TS}}
$$

to the transition state result, i.e.,

$$
\Delta F=\Delta F^{\mathrm{TS}}+\Delta F^{\mathrm{ad}} .
$$

As can be seen from Eq. (40), $\Delta F^{\text {ad }}$ scales with the ratio of conformational coordinates to the total number of degrees of freedom and, therefore, is usually small. It is always positive as a cunsequence of the enlarged phase space volume accessible due to the increased kinetic energy within the transition region.

\section{G. Relaxation correction to Eq. (26)}

We now consider the second aspect of the equilibrium approximation. For our transition state approach we assumed local equilibrium within the initial substate. This is certainly not the case immediately after the flooding potential has been switched on; rather, the system, perturbed by the flooding potential, will relax towards equilibrium. If the relaxation is as slow as the estimated MTT or even slower, the equilibrium assumption does not hold. This is expected to happen either for strong flooding or, more importantly, due to inherently slow relaxation processes. Employing a simple relaxation model we want to study how relaxation processes can modify our transition state estimate [Eq. (26)].

We return to the time-dependent conformation space density $\rho^{\mathbf{c}}(t, \mathbf{c})$ and assume that the flooding potential is switched on at $t=0$. For $t<0$ we have $\rho^{\mathbf{c}}(t, \mathbf{c})=\rho^{\mathbf{c}}(\mathbf{c})$. For $t \geq 0, \rho^{\mathbf{c}}(t, \mathbf{c})$ decays towards the new equilibrium density $\rho^{\mathbf{c}}(\mathbf{c}) \exp \left[-\beta V_{\mathrm{fl}}(\mathbf{c})\right]$, which is modified by the flooding potential. The time-dependent density implies a time-dependent partition function

$$
Z_{\mathbf{f l}}(t)=\int d^{m} c \rho^{\mathbf{c}}(t, \mathbf{c})
$$

(again, we dropped the contributions $Y$ from the momenta and from the fast degrees of freedom).

We describe the relaxation of $Z_{\mathrm{fl}}(t)$ as a multiexponential decay with $m$ relaxation times $\tau_{i}, i=$ $1, \ldots, m$. With that aim we assume that $Z_{\mathrm{fl}}(t) / Z_{0}$ [not necessarily $Z_{\mathrm{ff}}(t)$ alone] can be split into $m$ factors $\zeta_{i}(t)$,

$$
\frac{Z_{\mathrm{fl}}(t)}{Z_{0}}=\prod_{i=1}^{m} \zeta_{i}(t)
$$

each of which may be interpreted as describing one of the conformational degrees of freedom $c_{i}$. Each of the $\zeta_{i}$ is assumed to decay exponentially with a time constant $\tau_{i}$,

$$
\zeta_{i}(t)=\zeta_{i}(t \rightarrow \infty)+\left[\zeta_{i}(0)-\zeta_{i}(t \rightarrow \infty)\right] e^{-t / \tau_{i}} .
$$

The boundary values for the $\zeta_{i}$ are chosen canonically. At $t=0$ we have $Z_{\mathrm{fl}}(0)=Z_{0}$, thus we set $\zeta_{1}(0)=\cdots=$ $\zeta_{m}(0)=1$. Similarly, $Z_{\mathrm{fl}}(t \rightarrow \infty)=Z_{\mathrm{fl}}$ and, assuming equipartition for the destabilization free energy, we have $\zeta_{1}(t \rightarrow \infty)=\cdots=\zeta_{m}(t \rightarrow \infty)=\left(Z_{\mathrm{fl}} / Z_{0}\right)^{1 / m}$. Using Eq. (24) and these boundary values, Eq. (44) reads

$$
\zeta_{i}(t)=\left(\frac{Z_{0}}{\alpha}\right)^{1 / m}\left[1+\left(\alpha^{1 / m}-1\right) e^{-t / \tau_{i}}\right],
$$

which specifies our relaxation model.

To derive MTT's from that model we reconsider the right hand side of Eq. 20, which describes the decay of the probability $p(t)$, that the system is still within the initial substate at time $t \geq 0$,

$$
\frac{d p}{d t}=-\omega \kappa_{\mathrm{fl}} \frac{Z_{\mathrm{fl}}^{\neq}}{Z_{\mathrm{fl}}(t)} p(t),
$$

where $\kappa_{\mathrm{fl}}$ and $Z_{\mathrm{fl}}^{\neq}$are assumed to be constant. The integral of the above equation reads, using Eq. (17),

$$
p(t) \propto \exp \left\{-\frac{\alpha}{T_{0}} \int_{0}^{t} \frac{d t^{\prime}}{\prod_{i=1}^{m}\left[1+\left(\alpha^{1 / m}-1\right) e^{-t^{\prime} / \tau_{i}}\right]}\right\}
$$

and defines the MTT

$$
T_{\mathrm{fl}}=\frac{\int_{0}^{\infty} d t t p(t)}{\int_{0}^{\infty} d t p(t)}
$$

The integrals within the above two equations have to be solved numerically. We note that in the limit of fast relaxation $\left(\tau_{i} \ll T_{0} / \alpha\right.$ for all $\left.i\right) p(t)=\exp \left(-\alpha / T_{0}\right)$ and $T_{\mathrm{fl}}=T_{0} / \alpha$, which is the transition state result. On the other hand, if a number $m^{\prime}\left(m^{\prime} \leq m\right)$ of degrees of freedom relax slowly with respect to $T_{\mathrm{f}}$, the acceleration is decreased by at least the factor $\alpha^{m^{\prime} / m}$. Typically, one expects the $\tau_{i}$ 's to be distributed over a wide range of time scales. In this case, with increasing flooding strength and decreasing $T_{\mathrm{ff}}$, more and more $\tau_{i}$ 's will become slow with respect to $T_{\mathrm{fl}}$, and the increase of the acceleration factor will be reduced accordingly.

We summarize the nonequilibrium results. Our simple relaxation model suggested that slow relaxation processes within the system will decrease the acceleration factor even for weak flooding, whereas fast relaxation processes set an upper limit for the achievable acceleration. If the actual barrier crossing process is fast at the time scale of heat exchange between system and environment, an additional adiabatic correction is required, which scales linearly with the number $m$ of conformational coordinates used for flooding.

\section{TWO SAMPLE APPLICATIONS}

Two applications presented in the subsequent two sections are supposed to serve as examples and as test cases for our method. Both applications are computer experiments. In each case we compare transition products and transition rates obtained with $\mathrm{CF}$ with results from extended, unperturbed MD simulations. 


\section{A. Structural transitions in an argon cluster}

The first application is intended to test our estimates for the destabilization free energy of the flooding potential [Eqs. (26), (40), and (48)]. To that end, a large number of computer experiments is required to obtain good transition time statistics, and thus we had to use a simple system with few degrees of freedom. We have chosen a microcluster consisting of eight argon atoms with Lennard-Jones interactions [Fig. 5 (left)]. The free energy landscape of that system has been characterized elsewhere, and the lowest-energy minima are known (see, e.g., Ref. [62]).

\section{Methods}

The argon atoms were modeled as mass points $(m=40$ atomic units); their pairwise interaction is described by

$$
V_{i j}=4 E_{0}\left[\left(\frac{R}{r_{i j}}\right)^{12}-\left(\frac{R}{r_{i j}}\right)^{6}\right]
$$

where $r_{i j}$ is the distance between atoms $i$ and $j, E_{0}=$ $0.2464 \mathrm{kcal} / \mathrm{mol}$, and $R=3.42 \AA[63]$. The potential energy of the microcluster is

$$
V=\sum_{i<j} V_{i j}
$$

The system has been described classically, and the equations of motion were integrated using the Verlet algorithm [18] with an integration step size of $5 \mathrm{fs}$. The system was weakly coupled to a heat bath $(T=5 \mathrm{~K})$ with a coupling constant of $0.02 \mathrm{ps}^{-1}$ (as defined in Ref. [64]). Initial positions and velocities were obtained by equilibrating a compact random structure for $100 \mathrm{~ns}$. Fig. 5 (left) depicts that initial structure (in the figure van der Waals radii were shrunk, and nearest neighbors were connected by lines). That structure is identical to the conformation of lowest free energy given in Ref. [62].

We carried out $1500 \mathrm{MD}$ simulations with varying flooding parameter $E_{\mathrm{fl}}$, comprising a total simulation time of $35 \mu \mathrm{s}$. Since $V_{\mathrm{fl}}$ is not invariant under translations and rotations of the system [cf. Eq. (13)], the latter would "escape" the flooding potential with rigid body motion rather than CT's. Therefore these six degrees of
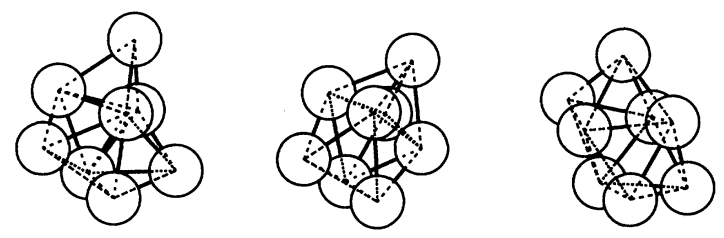

FIG. 5. Argon microclusters consisting of eight argon atoms each; the leftmost cluster was used as initial structure for the MD simulations described in the text; the other two are among those found with the flooding method; the lines connect nearest neighbors. freedom were removed in all simulations as described in Refs. [65,43].

The center $\overline{\mathbf{x}}$ and the covariance matrix $\mathbf{C}$ of the initial substate were computed according to Eqs. (9) and (8) from a subcanonical ensemble of 125000 structures derived from a MD simulation of $10 \mathrm{~ns}$ duration. In this simulation atomic positions were monitored and compared to the initial positions to ensure that no CT's occurred. Due to the elimination of rigid body motions, $\mathbf{C}$ has six vanishing eigenvalues and thus cannot be inverted, as required in Eq. (8). However, according to Eq. (13), $V_{\mathrm{fl}}(\mathbf{c})$ acts only in the subspace given by the $m$ eigenvectors of $\mathbf{C}$ with largest eigenvalues; hence $\mathbf{C}$ needs to be inverted only in that subspace which can be done.

To obtain essentially uncorrelated initial conditions for the 1500 flooding simulations, a set of 1500 structures and velocities was extracted at equidistant instances in time from the $10 \mathrm{~ns}$ MD simulation. For each of the flooding simulations initial positions and velocities were picked at random from this set. None of the initial conditions was used twice.

For each of the flooding simulations the time of escape from the initial substate was recorded. This was done by monitoring the multivariately scaled distance $d_{\boldsymbol{\Lambda}}(t)$ from the substate center of the system,

$$
d_{\mathbf{\Lambda}}(t):=\sqrt{\mathbf{c}(t)^{T} \mathbf{\Lambda}_{\mathrm{fl}} \mathbf{c}(t)},
$$

during the simulations. Abrupt changes of $d_{\boldsymbol{\Lambda}}$ to values larger than a critical limit $\Delta d$ were used as indicators of CT's. We have chosen $\Delta d=10 \AA$ on the basis of the following consideration. For Gaussian configuration space densities, $d_{\Lambda}^{2}(t)$ is $\chi^{2}$ distributed with $m=3 N-6=18$ degrees of freedom, with an expectation value of $4.18 \AA$ for $d_{\Lambda}$, and a standard deviation of $0.70 \AA$. Accordingly, distances that deviate from the expectation value by more than a few standard deviations should indicate that the system has left the substate. To exclude passages of the dividing hypersurface without subsequent transition we required $d_{\Lambda}(t) \geq 10 \AA$ for at least 20 ps (the latter value has been determined empirically).

\section{Results and discussion}

To illustrate the effect of the flooding potential, Fig. 6 shows a selection of three CT's out of the 1500 observed ones. They differ in the employed flooding strength per conformational coordinate, $\epsilon_{\mathrm{ff}}$. The figure depicts the time development of $d_{\boldsymbol{\Lambda}}(t)$ for each of the three flooding simulations. The estimates [based on Eqs. (26) and (40)] for the adiabatic destabilization free energy per degree of freedom, $\Delta f$, are given in brackets.

The observed transition times are shorter by factors of 706, 6200, and 56800 than the MTT for unperturbed CT's, which has been determined as $T_{0}=540$ ns (data not shown). Qualitatively, these examples agree with the expectation that CF should reduce MTT's. It is also apparent from the figure that each individual transition event is much faster than the relaxation with the heat bath, which was chosen as 50 ps. Thus our adiabatic treatment of the CT's is appropriate. 


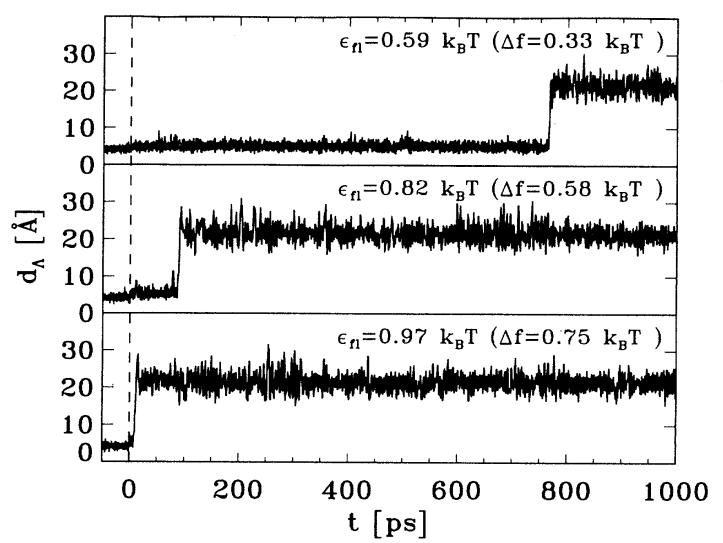

FIG. 6. CT's induced by a flooding potential of varying strength. The three curves show the multivariately scaled distance $d_{\Lambda}(t)$ as a function of time. At $t=0$ (dashed line) the flooding potential is switched on. Structural transitions are observed as jumps of $d_{\Lambda}$ after $765 \mathrm{ps}\left(\epsilon_{\mathrm{fl}}=0.59 k_{B} T\right)$, after $87 \mathrm{ps}\left(\epsilon_{\mathrm{fl}}=0.82 k_{B} T\right)$, and, almost instantaneously, after 9.5 ps $\left(\epsilon_{\mathrm{fl}}=0.97 k_{B} T\right)$, respectively.

Since the observed structural transitions are stochastic processes, individual transition times will deviate from their average. In order to check for quantitative agreement, we determined transition times for all 1500 flooding simulations. Figure 7 compares the results with our theory.

In the figure each dot represents one flooding simulation. The horizontal axis measures the adiabatic destabilization free energy for the flooding potential used in that particular simulation; the vertical position indicates the observed transition time in units of $T_{0}$ (logarithmic scale). The filled circles represent MTT averages of 50 simulations each; their size indicates the standard deviation (assuming a Poisson distribution for the transition times). The estimated transition time, according to the adiabatically corrected transition state estimate, is

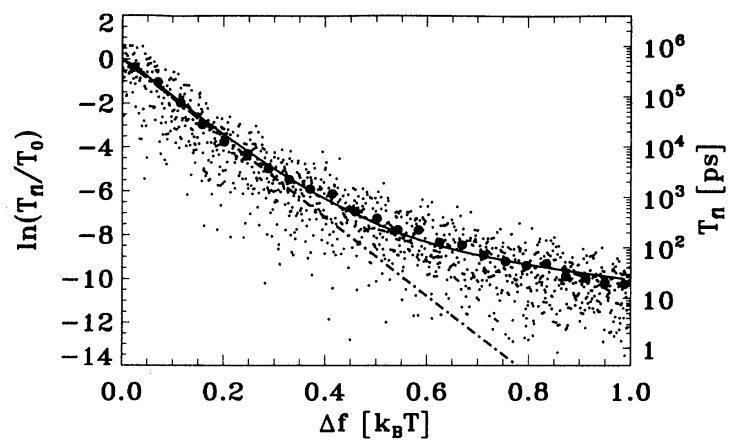

FIG. 7. Transition times (points) in units of $T_{0}$ (logarithmic scale) observed in 1500 flooding simulations with varying destabilization free energy $\Delta f$; the filled circles represent averaged transition times over 50 simulations each; the adiabatically corrected transition state estimate, according to Eqs. (26) and (40), is drawn as a dashed line; the solid line shows a fit of Eq. (48) to the data (see text). drawn as a dashed line.

As can be seen, average accelerations by factors up to 27000 can be achieved for this simple system. For weak flooding, the computer experiment agrees well with our transition state estimate for the acceleration factor. Admittedly, this is not too surprising, since for the small system at temperatures as low as $5 \mathrm{~K}$ the fluctuations of atomic positions are small, and thus the potential energy surface is nearly harmonic. As a result, $\rho^{\mathbf{c}}$ is Gaussian - which is the crucial assumption in our derivation of Eq. (26).

For larger flooding strength the acceleration factor falls below the transition state estimate. A fit (solid line in Fig. 7) of the nonequilibrium result [Eq. (48), with $p(t)$ given by Eq. (47)] to the observed MTT's shows that the data agree with the scenario of a hierarchy of relaxation times. For the fit, the $m$ relaxation times $\tau_{i}$ were assumed to be related to the eigenvalues $\lambda_{i}$ of the covariance matrix by

$$
\tau_{i}=\tau\left(\frac{\lambda_{i}}{\AA}\right)^{\mu}, \quad i=1, \ldots, m
$$

leaving $\tau$ and $\mu$ as two free parameters. Equation (52) is a generalization of the result for normal mode frequencies in harmonic systems derived by Gō [58] (there, $\mu=2$ ). Additionally, the shortest relaxation time, $\tau_{1}=0.7 \mathrm{ps}$, was derived from the relaxation time of the autocorrelation function of the atomic motions. The remaining undetermined parameter $\mu=3.5$ was derived from the fit, implying a slowest relaxation constant of $\tau_{m} \approx 20 \mathrm{~ns}$. Obviously, all relaxation times are much shorter than $T_{0}$, which explains the success of the transition state approach.

For larger irregular systems like proteins at higher temperatures, which we want to study with the flooding method, we expect slow relaxation processes. Also, $\rho^{\mathbf{c}}$ may be structured in quite a complex manner, and its description by a multivariate Gaussian may therefore be crude. To estimate transition rates in such cases, conventional free energy calculations have to be carried out, which require specifications of product states and of reaction coordinates. The question arises whether $\mathrm{CF}$ is robust enough to provide these prerequisites and to allow predictions of CT's.

\section{B. Conformational transitions in a simplified protein model}

The second set of computer experiments serves to address the above question. With that aim one would wish to choose as a test system an atomic protein model typically used in MD simulations and an appropriate structure determined from x-ray scattering experiments. Certainly one would expect that our method would induce some CT's; however, CT's of the unperturbed system would be too slow to be observed - so how should we convince ourselves that the induced CT's are the correct ones? 


\section{Methods}

We therefore decided to start with the simplified protein model described and studied in Ref. [15] which is known to exhibit fast CT's. The model is shown in Fig. 8 (left). It resembles a small protein of 100 amino acids length. In contrast to detailed MD models, in which each atom is considered explicitly, here each amino acid is represented by just one van der Waals sphere; hence the number of particles in the system is smaller by one order of magnitude than in more realistic protein models. The "residues" are linearly connected by interactions resembling chemical single bonds, as defined in the CHARMm force field [64]. Heterogeneous long ranged pair interactions between all residues model hydrophilic and hydrophobic forces of a solvent and stabilize the threedimensional structure of the model. As described in Ref. [15], that structure has been obtained from a simulated folding process. For further details we refer to Refs. $[15,49]$.

The dynamical properties of the simplified model have been shown to be qualitatively similar to those of the more realistic protein models with atomic resolution [49] with the desired exception that it exhibits relatively fast CT's at the time scale of one nanosecond. Three conformational states (depicted to the right in Fig. 8) have been identified in Ref. [15]. These properties qualify the model as an appropriate test system.

Unless otherwise specified, the flooding experiments have been carried out in a way similar to that described in the preceding subsection. The simulations have been performed at $300 \mathrm{~K}$ using an integration step size of $2 \mathrm{fs}$ for the numerical integration of the equations of motion.

We carried out 300 flooding simulations with varying flooding strength $E_{\mathrm{fl}}$. The total simulation time was 50 ns. A subcanonical ensemble of 31250 structures, derived from an $\mathrm{MD}$ simulation of 1 ns duration, was used to compute the flooding potential. From that ensemble we also extracted a set of 300 essentially uncorrelated initial conditions for the flooding simulations.

In contrast to the argon system, we selected only $m=$ 30 conformational coordinates to be affected by $V_{\mathrm{fl}}$, i.e.,
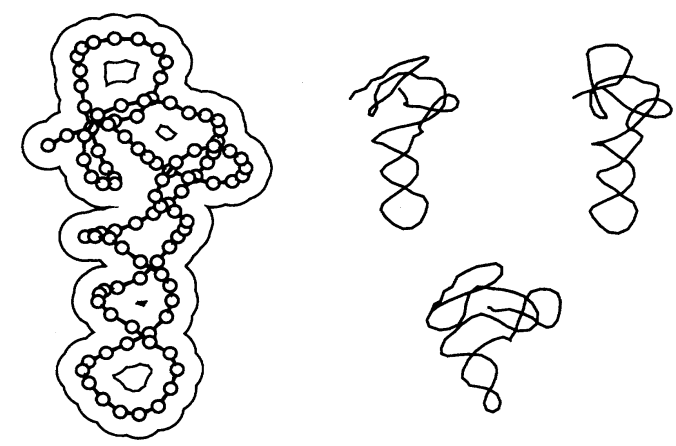

FIG. 8. Left: simplified protein model consisting of 100 van der Waals spheres, which are linearly connected by chemical bonds (lines); the van der Waals surface is indicated by the outer contour; right: three conformations are known [15]; these could be reproduced with CF.
$1 / 10$ of the total number of degrees of freedom. We based our choice on the observation that these 30 degrees of freedom contribute $82 \%$ to the total mean squared atomic fluctuations within the initial substate and, therefore, are expected to dominate the transitions.

Again, a multivariately scaled distance $d_{\boldsymbol{\Lambda}}(t)$ from the substate center [Eq. (51)] was used to detect CT's. The appropriate $\chi^{2}$ distribution with $m=30$ degrees of freedom has an expectation value of $5.4 \AA$ and a standard deviation of $0.70 \AA$; accordingly we used sudden distance changes of more than $10 \AA$ as an indicator of CT's. A CT was accepted if $d_{\boldsymbol{\Lambda}}$ did not drop below $10 \AA$ for 20 ps. In cases where $d_{\boldsymbol{\Lambda}}$ did not provide clear transition times we additionally inspected projections of the trajectory $\mathbf{x}(t)$ onto the plane defined by the two largest eigenvectors of the covariance matrix $\mathbf{C}$.

\section{Results and discussion}

Figure 9 shows the 300 observed transition times (dots) in units of $T_{0}=1404 \mathrm{ps}$ on a logarithmic scale. As in Fig. 7 the abscissa measures $\Delta f$ in units of $k_{B} T$. The filled circles represent MTT's, which have been obtained by averaging 10 transition times each.

The experiment shows that also the complex CT's depicted in Fig. 8 can be accelerated by at least two orders of magnitude. Among the six product CS's found in the flooding simulations were the three known from unperturbed simulations (rms deviation of the structures below $1 \AA$ ). The other three are new; they have not been observed in a simulation of $100 \mathrm{~ns}$ duration [43]. We checked the stability of each of the 300 product states by switching off the flooding potential 5 ps after the CT and by subsequently monitoring structural deviations for another 10 ps. More than $92 \%$ of the product states turned out to be stable; these showed rms deviation below $1 \AA$ (data not shown). In summary, most of the simulations predicted CT's which have been observed or are likely

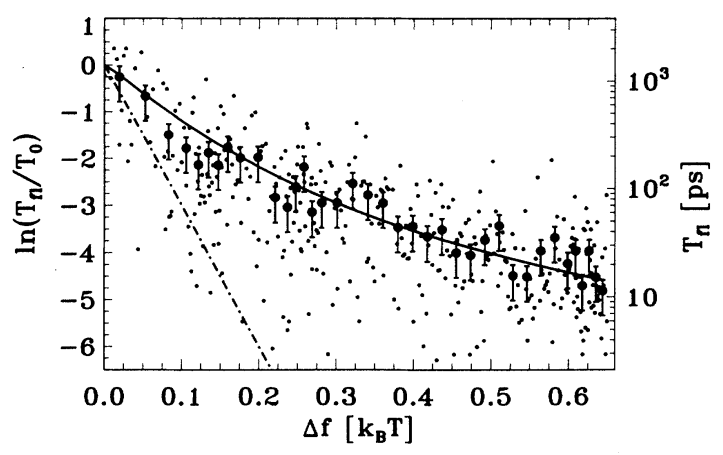

FIG. 9. Transition times (points) in units of $T_{0}$ (logarithmic scale) observed in 300 flooding simulations with varying destabilization free energy $\Delta f$; the filled circles represent averaged transition times over ten simulations each; the error bars show the corresponding standard deviations; the transition state estimate [Eq. (26)] is drawn as a dashed line; the solid line shows a fit of the nonequilibrium result [Eqs. (48) and (47)]. 
to be observed in unperturbed MD simulations. In this sense, our method predicted correct CT's. This is the main result of this section.

In contrast to the simple argon system the transition state estimate (dashed line in Fig. 9) for the acceleration factor fails even for weak flooding. Recalling our assumptions, this failure is either due to slow relaxation processes or due to a complex, non-Gaussian structure of $\rho_{k}^{\mathbf{c}}$, or both. While it appears to be difficult to detect significant deviations from high-dimensional Gaussian distributions with limited available data, the nonequilibrium estimate allows one to check whether a simple relaxation model can explain the observed transition times.

To that aim we fitted Eq. (48) to the MTT's (solid line in Fig. 9). Again, we used Eq. (52) to relate the 30 relaxation constants $\tau_{i}$ to the atomic fluctuations $\lambda_{i}$, and we determined the shortest relaxation time, $\tau_{1}=0.7 \mathrm{ps}$, from the autocorrelation function of the corresponding conformational degree of freedom [43]. From the fit we obtained $9<\mu<11$. With these parameters, 12 of the 30 relaxation times are shorter than $1 \mathrm{~ns}, 7$ lie between $1 \mathrm{~ns}$ and $1 \mu \mathrm{s}$, while 11 relaxation times are longer (partially much longer) than $1 \mu \mathrm{s}$.

Since the fit turned out to be quite unstable, and because it is based on inaccurate MTT's, care has to be taken in the interpretation of this result. In particular, we can only give lower bounds for the long relaxation times, which almost diverge in the fit. Despite these uncertainties the analysis shows that the simple relaxation model is indeed consistent with our computer experiments if (a) the $\tau_{i}$ are assumed to cover a wide range of time scales and if (b) a significant fraction of the $\tau_{i}$ are larger than $T_{0}$. In that respect our model study captured prominent features of proteins and glasses.

We recall that we did not study the influence of a probably non-Gaussian conformation space density, which is also expected to contribute to the observed limitation of the acceleration factor.

\section{SUMMARY AND CONCLUSION}

Based on a coarse-grained model for the conformation space density, which describes conformational substates (CS's) of proteins and other heterogeneous macromolecular systems, we derived a method to accelerate conformational transitions (CT's) out of a given CS. Application of the method, which we termed "conformational flooding" (CF), involves two steps. First, the density model is computed from a usually short molecular dynamics (MD) simulation. From that model an artificial flooding potential is derived, included into the Hamiltonian of the system, and a second simulation is carried out. The flooding potential accelerates CT's, which, therefore, are likely to be observed during the "flooding simulation." We gave analytical estimates for the acceleration factor.

We provided two sample applications. First we considered a small argon microcluster at low temperature. For that system the coarse-grained density model was assumed to be a good approximation. Indeed, the observed acceleration for varying flooding strength agreed well with the analytical estimate, and known low-energy structures were found. This computer experiment thus confirmed the feasibility of the method for that simple system.

In the second application we considered the much more complex collective transitions observed in proteins, in which slow structural relaxations are known to occur, and where the subcanonical configuration space density may be non-Gaussian: Despite these complications the flooding method proved sufficiently robust to accelerate also CT's of the protein model by more than two orders of magnitude. Still, the method predicted correct transitions in $92 \%$ of the 300 simulations carried out.

A simple relaxation model suggested that the achievable acceleration of CT's is limited mainly by the fastest relaxation processes within the system. Based on this finding we expect our method to bring structural transitions as slow as few microseconds into the scope of MD simulations for a wide variety of complex systems.

Several possible generalizations of the CF method come into mind. First, more accurate conformation space density models may be used. These can be constructed by methods of nonlinear statistical data analysis such as vector quantization techniques [66-68]. In contrast, if large numbers of CS's are to be studied, e.g., for simulations of protein unfolding, further approximations to the conformation space density are desirable. With that aim, groups of substates may be clustered and - if needed, recursively - approximated by one single, larger multivariate Gaussian. Finally, computational costs can be reduced by excluding those parts of a system from flooding, which can be assumed a priori not to take part actively in the CT under consideration. This is most likely the case, e.g., for solvent molecules or solvent-exposed sidegroups of a protein. In a similar manner, among atoms which exhibit strongly correlated motion only a small fraction may be selected for flooding.

Applications of the flooding method are abundant; we sketch a few.

(i) Structure determination. Incomplete experimental data on protein structure require one to search conformation space for those conformations with low free energy, which agree with the experimental data. Conventionally, MD simulations are used for that purpose [69]. Our method helps to escape from local minima in that process.

(ii) Stability of protein models. A well-established check of a given protein model is to observe its deviation from the experimental structure during a MD simulation; large deviations indicate an unstable and hence unrealistic model. Unfortunately, severe deviations may develop too slowly to be detected in a conventional MD simulation, which makes the check somewhat unreliable. Conformational flooding provides a more rigorous test.

(iii) Conformation search. When iteratively applied, CF serves to explore configuration space. The method can thus be used to find low-energy conformers as well as to characterize the distribution of CS's in configuration space. An example is given in Ref. [70].

(iv) Prediction of functional motions. Similarly, reactions involving not only one but a sequence of CT's, e.g., allosteric interactions or unfolding processes, can be described and - depending on the complexity and quality 
of the used molecular model - also predicted.

(v) Estimation of thermodynamic quantities. Finally, methods to estimate free energies, entropies, or partition functions [56,57] can be combined with CF to improve the estimates by taking larger regions of phase space into account.

The flooding method has been implemented into the parallelized MD code EGO_VIII and is available from the authors [71].

\section{ACKNOWLEDGMENTS}

The author wishes to thank Paul Tavan and Helmut Heller for inspiring discussions and for carefully reading the manuscript. This work has been supported by the Deutsche Forschungsgemeinschaft (SFB 143/C1) and the Deutscher Akademischer Austauschdienst (PROCOPE/Tavan).
[1] G. U. Nienhaus, J. R. Mourant, and H. Frauenfelder, Proc. Natl. Acad. Sci. U.S.A. 89, 2902 (1992).

[2] W. Köhler, J. Friedrich, and H. Scheer, Phys. Rev. A 32, 660 (1988).

[3] I. E. T. Iben et al., Phys. Rev. Lett. 62, 1916 (1989).

[4] B. A. Wallace, Annu. Rev. Biophys. Biophys. Chem. 19, 127 (1990).

[5] L. Stryer, Biochemistry (W. H. Freeman and Company, San Francisco, 1988).

[6] K. P. Hofmann, in GTPases in Biology; Handbook of Experimental Pharmacology, edited by B. Dickey and L. Birnbaumer (Springer, Berlin, 1995), pp. 955-978.

[7] T. E. Creighton, Biochem. J. 270, 1 (1990).

[8] F. E. Cohen et al., Science 264, 530 (1994).

[9] H. Frauenfelder, G. A. Petsko, and D. Tsernoglou, Nature (London) 280, 558 (1979).

[10] R. H. Austin et al., Biochem. 14, 5355 (1975).

[11] R. Elber and M. Karplus, Science 235, 318 (1987).

[12] N. Gō and T. Noguti, Chem. Scr. 29A, 151 (1989).

[13] E. Shakhnovich, G. Farztdinov, A. M. Gutin, and M. Karplus, Phys. Rev. Lett. 67, 1665 (1991).

[14] A. E. García, Phys. Rev. Lett. 68, 2696 (1992).

[15] H. Grubmüller and P. Tavan, J. Chem. Phys. 101, 5047 (1994).

[16] H. Frauenfelder, S. G. Sligar, and P. G. Wolynes, Science 254, 1598 (1991).

[17] J. A. McCammon and M. Karplus, Proc. Natl. Acad. Sci. U.S.A. 76, 3585 (1979).

[18] L. Verlet, Phys. Rev. 159, 98 (1967).

[19] W. F. van Gunsteren and H. J. C. Berendsen, Angew. Chem. Int. Ed. Engl. 29, 992 (1990).

[20] K. Müller and L. D. Brown, Theor. Chim. Acta 53, 75 (1979).

[21] C. J. Cerjan and W. H. Miller, J. Chem. Phys. 75, 2800 (1981).

[22] K. D. Gibson and H. A. Scheraga, J. Comput. Chem. 11, 468 (1990).

[23] S. Fischer and M. Karplus, Chem. Phys. Lett. 194, 252 (1992).

[24] J. Cao and B. J. Berne, J. Chem. Phys. 92, 1980 (1990).

[25] S. E. Huston and G. R. Marshall, Biopolymers 34, 75 (1994).

[26] R. Khare, T. Lazaridis, and M. E. Paulaitis, Chem. Des. Autom. News 8, 28 (1993).

[27] P. Hänggi, P. Talkner, and M. Borkovec, Rev. Mod. Phys. 62, 251 (1990).

[28] R. Elber, in New Developments in Theoretical Studies of Proteins, edited by R. Elber (World Scientific, Singapore, in press).

[29] O. S. Smart, Chem. Phys. Lett. 222, 503 (1994)
[30] R. Czerminski and R. Elber, Int. J. Quantum. Chem. 24, 167 (1990).

[31] J. Schlitter, M. Engels, and P. Krüger, J. Mol. Graphics 12, 84 (1994).

[32] M. A. Ech-Cherif El-Kettani and J. Durup, Biopolymers 32, 561 (1992).

[33] S. C. Harvey and H. A. Gabb, Biopolymers 33, 1167 (1993).

[34] D. Chandler, J. Chem. Phys. 68, 2959 (1978).

[35] J. P. Valleau and G. M. Torrie, in Statistical Mechanics Part A: Equilibrium Techniques, edited by B. J. Berne (Plenum Press, New York, 1977), p. 137.

[36] J. A. McQuarrie, Statistical Mechanics (Harper \& Row, New York, 1975).

[37] R. Elber, J. Chem. Phys. 93, 4312 (1990).

[38] J. Schlitter and H.-J. Steinhoff (unpublished).

[39] R. E. Bruccoleri and M. Karplus, Biopolymers 29, 1847 (1990).

[40] J. P. Stoessel and P. Nowak, Macromolecules 23, 1961 (1990).

[41] A. Ansari et al., Biophys. Chem. 26, 337 (1987).

[42] H. C. Anderson, J. Chem. Phys. 72, 2384 (1980).

[43] H. Grubmüller, N. Ehrenhofer, and P. Tavan, J. Chem. Phys. (to be published).

[44] F. Zhou, A. Windemuth, and K. Schulten, Biochem. 32, 2291 (1993)

[45] G. Váró and J. K. Lanyi, Biochem. 30, 5016 (1991).

[46] H.-J. Steinhoff, J. Schlitter, A. Redhardt, and D. Husmeier, Biochim. Biophys. Acta 1121, 189 (1992).

[47] N. Gō and H. A. Scheraga, J. Chem. Phys. 51, 4751 (1969).

[48] H. Frauenfelder, P. J. Steinbach, and R. D. Young, Chem. Scr. 29A, 145 (1989).

[49] H. Grubmüller, Ph.D. thesis, Technische Universität München, 1994.

[50] A. Amadei, A. B. M. Linssen, and H. J. C. Berendsen, Proteins 17, 412 (1993).

[51] C. Levinthal, J. Chem. Phys. 65, 44 (1968).

[52] C. W. Gardiner, Handbook of Stochastic Methods (Springer-Verlag, Berlin, 1985).

[53] B. J. Berne, Multiple Time Scales, 1st ed. (Academic Press, Orlando, Florida, 1985), Chap. 13, pp. 419-436.

[54] K. G. Wilson, Rev. Mod. Phys. 47, 773 (1975).

[55] D. Shalloway, J. Global Opt. 2, 281 (1992).

[56] M. Karplus and J. N. Kushick, Macromolecules 14, 325 (1981).

[57] J. Schlitter, Chem. Phys. Lett. 215, 617 (1993).

[58] N. Gō, Biophys. Chem. 35, 105 (1990).

[59] S. Hayward, A. Kitao, and N. Gō, Phys. Scr. 3, 936 (1994). 
[60] H. Eyring, J. Chem. Phys. 3, 107 (1935).

[61] H. A. Kramers, Physica (Utrecht) VII, 284 (1940).

[62] M. Orešič and D. Shalloway, J. Chem. Phys. 101, 9844 (1994).

[63] P. W. Atkins, Physical Chemistry (Oxford University Press, Oxford, 1990).

[64] B. R. Brooks et al., J. Comput. Chem. 4, 187 (1983).

[65] C. Eckart, Phys. Rev. 47, 552 (1935).

[66] P. Tavan, H. Grubmüller, and H. Kühnel, Biol. Cybern. 64, 95 (1990).

[67] K. Rose, E. Gurewitz, and G. Fox, Phys. Rev. Lett. 65,
945 (1990).

[68] C. Hillermeier, N. Kunstmann, B. Rabus, and P. Tavan, Biol. Cybern. 72, 103 (1994).

[69] A. T. Brünger, J. Kuriyan, and M. Karplus, Science 235, 458 (1987).

[70] H. Grubmüller, Phys. Rev. Lett. (to be published).

[71] M. Eichinger, H. Grubmüller, and H. Heller, "User Manual for EGO_VIII, Release 1.0," Arbeitsgruppe Theoretische Biophysik am Institut für Medizinische Optik der Universität München, Theresienstr. 37, 80333 München, Germany, 1995. 